\title{
The market for migraine drugs
}

\author{
Angela M. Sparrow* and Jonathan W. Searles
}

Migraine is a highly prevalent disease, affecting more than 33 million people in the US alone, with three times greater prevalence in females. Migraine pain is often debilitating. Individual attacks last for 4-72 hours, often accompanied by nausea, vomiting, photophobia and/or phonophobia.

The market for prescription acute and preventive migraine treatments has been stable - and largely generic for many years, but now stands poised for transformation. 2018 witnessed the launch of the first novel preventive agents since 2010 and additional preventive and acute agents are expected to launch in the next 5 years. Amongst these high-cost, innovative new drugs, seven or more therapies target calcitonin gene-related peptide (CGRP) a key pathological factor in migraine and a sought-after, but once elusive, drug target. These comprise the first new migrainespecific class of drugs to launch in more than two decades.

\section{Current treatment}

There are two main clinical subtypes of migraine based on headache frequency: episodic migraine (EM), in which patients have $<15$ headache days per month, and chronic migraine (CM), in which patients have $\geq 15$ headache days per month, with at least 8 of those days having migrainous features. Migraine treatment is divided into two paradigms: acute treatment, which is taken as an abortive treatment at the onset of an attack, and prophylactic treatment, which is administered chronically to prevent new migraine attacks. Acutely, many migraineurs treat - sometimes exclusively - with over-the-counter medications, and some patients also rely on prescription analgesics. The triptans (named for the ending of the molecule names) are the mainstays of prescription acute migraine treatment. Sumatriptan launched in the early 1990s, followed by six additional triptans, which together revolutionized the acute migraine market. Since then, additional delivery routes have offered largely incremental benefits. Novel non-triptan alternatives are needed for patients for whom triptans are contraindicated or who do not respond to or cannot tolerate them.
The most commonly prescribed drugs for migraine prevention are antiepileptic drugs (such as topiramate), beta blockers (such as propranolol) and tricyclic antidepressants (including amitriptyline), some of which have migraine prevention on their labels. Unfortunately, many of these agents have side effects including cognitive problems, fatigue, somnolence, weight gain and, particularly concerning in a largely female population, a risk of teratogenic effects. Until 2018, the last meaningful innovation in this arena was the approval of Botox for CM in 2010. Botox is the only nondaily injectable preventive therapy, has far fewer side effects than daily oral agents and is the only therapy specifically approved for CM (clinical trial data do not support its use in patients with EM).

Despite the array of available, mostly inexpensive options, current prophylactics leave much to be desired in terms of efficacy; response rates fall well short of $100 \%$ and breakthrough attacks are common, as is polypharmacy to achieve maximal results. Moreover, because of side effects, compliance and adherence are often low.

The (re)birth of CGRP-targeted therapies CGRP is a potent vasodilatory neuropeptide. Its release produces local vasodilation and extravasation of plasma and plasma proteins into surrounding tissue. Basal levels of peripheral CGRP are higher in patients with CM than in patients with EM or healthy controls; intravenous administration of CGRP can trigger migraines in predisposed patients. In the early 2000s, numerous oral CGRP antagonists were being developed for migraine. The leading candidate at the time was Merck \& Co.s telcagepant; however, hepatotoxicity issues surfaced in late-phase trials and Merck discontinued their CGRP programme. Other developers followed suit.

Since then, there has been a resurgence of interest in the class (TABLE 1). Several monoclonal antibodies (mAbs) that target CGRP or its receptor (hereafter collectively referred to as anti-CGRP $\mathrm{mAbs}$ ) are in development or have recently been approved for migraine prevention: erenumab (Aimovig; Amgen/Novartis), fremanezumab

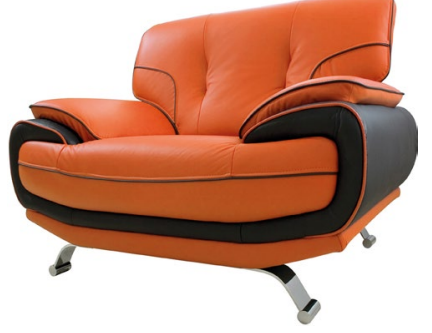

(Ajovy; Teva), galcanezumab (Emgality; Eli Lilly) - which all reached the US market in 2018 (European launches soon to follow) - and Alder BioPharmaceuticals' eptinezumab (phase III). Though far from a cure, reported clinical trial data have stirred excitement. Topiramate and Botox produce a 1-2 day reduction in monthly migraine days in patients with $\mathrm{CM}$, with a response rate $(\geq 50 \%$ reduction in monthly migraine days) of $12 \%$ for Botox and $22 \%$ for topiramate. In comparison, in phase III data, the anti-CGRP mAbs resulted in an approximately 1.5-2 day reduction in monthly migraine days in patients with EM and a 2-2.5 day reduction in patients with $\mathrm{CM}$; the response rate in both populations was $12-24 \%$. Although these results are similar to those for topiramate and Botox (based on cross-trial comparison), in secondary end point analysis a portion of patients treated with anti-CGRP mAbs experience a $75-100 \%$ reduction in monthly migraine days, patients administer fewer doses of acute drugs, and some patients

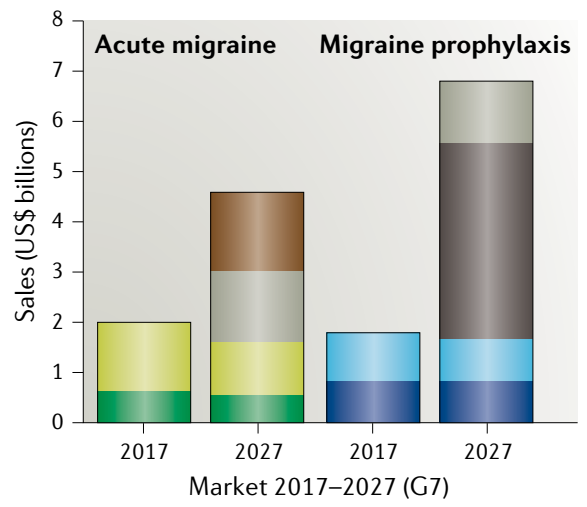

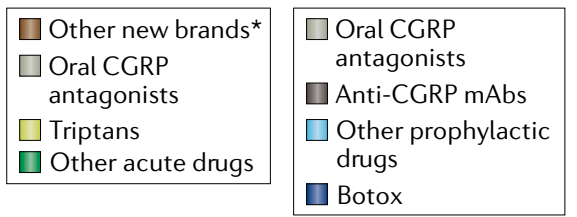

Fig. 1 | Sales and predicted sales of key drug classes in $\mathbf{G 7}$ countries for migraine, by market segment. The G7 countries are the US, France, Germany, Italy, Spain, UK and Japan. *Other new brands include Eli Lilly's lasmiditan and several reformulations of existing drugs. CGRP, calcitonin gene-related peptide, mAbs, monoclonal antibodies. Source: Decision Resources Group. 
Table 1 | Selected CGRP-targeted therapies in development for migraine

\begin{tabular}{|c|c|c|c|c|}
\hline Drug & Developer & Mechanism of action & $\begin{array}{l}\text { Market } \\
\text { segment }\end{array}$ & Status \\
\hline $\begin{array}{l}\text { Erenumab } \\
\text { (Aimovig) }\end{array}$ & Amgen/Novartis & $\begin{array}{l}\text { Fully human mAb } \\
\text { targeting the CGRP } \\
\text { receptor }\end{array}$ & Prophylaxis & Marketed (US) \\
\hline $\begin{array}{l}\text { Fremanezumab } \\
\text { (Ajovy) }\end{array}$ & Teva & $\begin{array}{l}\text { Humanized mAb } \\
\text { targeting CGRP }\end{array}$ & Prophylaxis & Marketed (US) \\
\hline $\begin{array}{l}\text { Galcanezumab } \\
\text { (Emgality) }\end{array}$ & Eli Lilly & $\begin{array}{l}\text { Humanized mAb } \\
\text { targeting CGRP }\end{array}$ & Prophylaxis & Marketed (US) \\
\hline Eptinezumab & $\begin{array}{l}\text { Alder } \\
\text { BioPharmaceuticals }\end{array}$ & $\begin{array}{l}\text { Humanized mAb } \\
\text { targeting CGRP }\end{array}$ & Prophylaxis & Phase III \\
\hline Atogepant & Allergan & $\begin{array}{l}\text { CGRP receptor } \\
\text { antagonist }\end{array}$ & Prophylaxis & Phase III \\
\hline Rimegepant & Biohaven & $\begin{array}{l}\text { CGRP receptor } \\
\text { antagonist }\end{array}$ & Prophylaxis & Phase III \\
\hline Ubrogepant & Allergan & $\begin{array}{l}\text { CGRP receptor } \\
\text { antagonist }\end{array}$ & Prophylaxis & Phase III \\
\hline BHV-3500 & Biohaven & $\begin{array}{l}\text { CGRP receptor } \\
\text { antagonist }\end{array}$ & Prophylaxis & Phase I \\
\hline
\end{tabular}

CGRP, calcitonin gene-related peptide; mAb, monoclonal antibody.

report that pain severity is decreased and breakthrough migraines are more manageable. Importantly, erenumab is effective in patients who have failed prior preventive treatment either for efficacy or tolerability reasons. Another key feature is the excellent tolerability and safety of these drugs; indeed, the FDA labels for erenumab, fremanezumab and galcanezumab are free of warnings and precautions (aside from hypersensitivity) and the adverse events are largely confined to injection site reactions.

All four mAbs have similar efficacy and tolerability data, so differentiation is critical. In addition to a short (a few months) first-to-market advantage, erenumab is also the only fully human $\mathrm{mAb}$ and targets the CGRP receptor rather than CGRP itself. The direct clinical benefits of this are unclear, but this distinction could support preferential prescribing of erenumab to patients who do not respond to a $\mathrm{mAb}$ that targets CGRP itself. The other main differences are in delivery: erenumab and galcanezumab are offered as once-monthly, at-home subcutaneous (s.c.) injections via prefilled autoinjectors; fremanezumab, also a s.c. injection, is available only in prefilled syringes, but can be dosed monthly or quarterly. Eptinezumab will be administered as a once-quarterly intravenous infusion. Access and contract negotiation with payers will also be key drivers in treatment selection. In the US, payers are still finalizing their coverage decisions for 2019 , and it is unlikely that any provider will include all three mAbs - as seen recently with ExpressScripts' decision to exclude fremanezumab from its 2019 national preferred formulary.

Oral CGRP-targeted therapies are also in development. In the post-telcagepant era, Allergan and Biohaven acquired the rights to CGRP antagonists from Merck (ubrogepant) and Bristol-Myers Squibb (rimegepant), respectively; both companies believe that liver toxicity observed with telcagepant is not a class effect. Phase III trials for the acute treatment of migraine are underway. Available efficacy data suggest the response rates may be lower than those of triptans (cross-trial comparisons), but the safety and tolerability data are favourable, with no elevations in liver enzymes. In the prevention space, Allergan is developing atogepant, another Merck cast-off, currently in phase III development. In late 2018, Biohaven initiated clinical trials for rimegepant for migraine prophylaxis and has another phase I candidate, BHV-3500. Although little is known about the efficacy of these drugs, patients often prefer the oral route of administration to s.c. injection. However, because the mAbs are infrequently dosed, efficacy will be the deciding factor for many patients, especially those with severe or frequent migraines.

\section{Market indicators}

The 2017 migraine market is estimated at US $\$ 3.8$ billion in the seven major markets (US, France, Germany, Italy, Spain, UK and Japan), with the acute market accounting for just over half of that (FIG. 1). However, the market is primed for dramatic growth over the next 10 years, potentially tripling in size to over $\$ 11$ billion, spurred by CGRP-targeted drugs, which could account for more than $\$ 6.5$ billion of sales in 2027.

After much anticipation, erenumab launched in the US market in May 2018 , followed by fremanezumab and galcanezumab at the end of Q3 2018. Erenumab has also received European Medicines Agency approval whereas fremanezumab and galcanezumab are both under review. Eptinezumab is expected to launch in the US in 2020. Given the cost of these therapies (approximately $\$ 7,000$ per year in the US), prescribing will almost certainly be limited to patients who have failed prior treatments; however, even a small uptake will result in considerable revenue owing to the enormous population size. Initial indicators point to rapid, early adoption of anti-CGRP mAbs in the US, likely due to pent-up demand for improved options. Combined with the anticipated launch of atogepant in 2022, the migraine prophylaxis market could nearly quadruple to $\$ 7$ billion by 2027 .

In the acute space, migraine experts estimate that $15-20 \%$ of patients are candidates for non-triptan alternatives because of intolerability, ineffectiveness or contraindications. Ubrogepant and rimegepant are expected to launch in the US in 2020 (the timing of ex-US launches remains unclear owing to the absence of global trials). In addition, Eli Lilly's lasmiditan, a novel 5-hydroxytryptamine $1 \mathrm{~F}$ $\left(5-\mathrm{HT}_{1 \mathrm{~F}}\right)$ receptor agonist (phase III), could add to the crop of migraine-specific, nontriptan options. However, as for the mAbs, all three of these drugs will enter a heavily generic market, relegating them to secondline or later-line treatment. Nevertheless, the acute migraine market, driven by the oral CGRP antagonists, could more than double to more than $\$ 4$ billion by 2027 . Payers could soon be bracing for the impact of CGRP-targeted therapies in the acute realm, too.

$$
\begin{array}{r}
\text { Angela M. Sparrow* and Jonathan W. Searles } \\
\text { Decision Resources Group, Burlington, } \\
\text { Burlington, MA, USA. } \\
\text { *e-mail: asparrow@TeamDRG.com }
\end{array}
$$

Competing interests

The authors declare no competing interests. 